\title{
Suchmaschinen auf dem Prüfstand - eine vergleichende Inhaltsanalyse der Qualität von Trefferlisten
}

Melanie Magin / Miriam Steiner / Dominique Heinbach / Sarah Bosold / Alice Pieper / Eva-Maria Felka / Birgit Stark

Suchmaschinen sind für die Nutzer ein zentrales Werkzeng zur Erschließung des Internets. Als Intermediäre filtern sie die Informationsflut im Netz und identifizieren bedeutsame Inbalte, haben also eine ähnliche Funktion und eine vergleichbare gesellschaftliche Verantwortung wie der Journalismus. Damit stellt sich die Frage nach der Qualität ibrer Trefferlisten, die in der Kommunikationswissenschaft bislang kaum erforscht wurde. Die vorliegende Studie untersucht dem Ansatz der nutzerzentrierten Inhaltsanalyse folgend die Benutzerfreundlichkeit, Relevanz, Gattungsvielfalt und Aktualität der Trefferlisten von fünf Suchmaschinen zu sechs gesellschaftlich relevanten, kontroversen Themen. Auf diese Weise wird geprüft, ob die Suchmaschinen die Voraussetzungen dafür bieten, dass die Nutzer zwei Rollen erfüllen können: die des Konsumenten, für den der Nutzwert im Fokus steht, und die des Bürgers, der sich an gesellschaftlichen Wertvorstellungen orientiert. Die Ergebnisse zeigen, dass Google insgesamt qualitativ hochwertigere Trefferlisten bietet als die Mitbewerber Bing, Ixquick, Lycos und Ask, binsichtlich der Gattungsvielfalt aber etwas hinter der Konkurrenz zurückbleibt.

Schlüsselwörter: Suchmaschinen, Qualität, Inhaltsanalyse, Trefferlisten, Benutzerfreundlichkeit, Relevanz, Vielfalt, Aktualität

\section{Einleitung}

In jüngster Zeit wurde Google wiederholt Gegenstand öffentlicher Diskussionen. Im Mai 2014 forderte der deutsche Wirtschaftsminister Sigmar Gabriel das Bundeskartellamt auf zu prüfen, ob das Unternehmen seine marktbeherrschende Stellung missbrauche, um Konkurrenten systematisch zu verdrängen. Im November 2014 plädierte das EUParlament in einer (allerdings nicht bindenden) Resolution für „eine Entflechtung von Suchmaschinen von anderen ,kommerziellen Dienstleistungen“" (Heuzeroth 2014). Die Angriffe richten sich gegen eines der kapitalstärksten Unternehmen der Welt mit einem weltweiten Umsatz von rund 66 Milliarden USD und einem Nettogewinn von mehr als 14 Milliarden USD im Jahr 2014 (Wäscher 2015). In Deutschland ist Googles Marktanteil mit über 90 Prozent im internationalen Vergleich besonders hoch (Latzer et al. 2014, S. 12).

Die Sorgen, die in den Diskussionen zum Ausdruck kommen, hängen mit der Schlüsselstellung von Suchmaschinen im Internet zusammen. Als Informationsintermediäre und Gatekeeper ${ }^{1}$ regeln sie den Zugang zu Internet-Inhalten (Latzer et al. 2014, S. 6).

1 Suchmaschinen als Gatekeeper zu betrachten, ist nicht unumstritten. Röhle (2010, S. 29) verweist darauf, dass Suchmaschinen, anders als Journalisten, selbst keine Themen auf die Agenda setzen, keine homogene Nachrichtenauswahl produzieren, und es viele alternative Möglichkeiten der Informationsbeschaffung gebe. Zentral für die hier betrachtete Fragestellung ist aber, dass Suchmaschinen für die Nutzer die „Weichen“ für den Zugang ins Netz stellen, was im Bild des Gatekeepers treffend zum Ausdruck kommt. 
Was in ihren Trefferlisten nicht auftaucht, nimmt die Mehrheit der Nutzer nicht wahr. Damit „besteht die begründete Gefahr, dass man nicht mehr ,findet, was man sucht', sondern, dass man das findet, wovon Google möchte, dass es gefunden wird. (...) Zentral ist nicht mehr die Frage nach den rezipierten Inhalten, sondern nach dem Auswahlprozess derselben durch den Intermediär“ (Dörr \& Schuster 2014, S. 300). Wie dieser Auswahlprozess abläuft, ist jedoch weitgehend unbekannt. Die Kriterien, nach denen ihre Algorithmen Treffer anzeigen und positionieren, sind streng gehütete Firmengeheimnisse und ändern sich fortwährend. Bekannt sind nur die wichtigsten, etwa die zentrale Bedeutung der Linkstruktur für Googles Page Rank - und viele Nutzer wissen selbst darüber nicht Bescheid (Stark et al. 2014). Hinzu kommt, dass Suchmaschinen sich vorrangig an ökonomischen Maximen orientieren statt an ihrer gesellschaftlichen Verantwortung, die sie durch ihre Gatekeeper-Funktion innehaben, an die sie im Gegensatz zu den Massenmedien aber nicht per Gesetz gebunden sind (Stark 2014, S. 4).

Umso wichtiger ist es, nach der Qualität ihrer Trefferlisten zu fragen. Anders als mit der Qualität journalistischer Angebote (Arnold 2009; Neuberger 2012) hat sich die Kommunikationswissenschaft mit der Qualität von Suchmaschinen bisher kaum befasst. Die wenigen vorhandenen Inhaltsanalysen der Trefferlisten von Universalsuchmaschinen sind älteren Datums (Machill et al. 2003, S. 98; Neuberger 2005), und ihre Befunde dürften aufgrund der rasanten Weiterentwicklungen auf dem Suchmaschinenmarkt kaum auf die heutige Situation übertragbar sein. In der einzigen aktuellen Inhaltsanalyse von Trefferlisten untersucht Beiler (2013) die Qualität von Nachrichtensuchmaschinen, er beschränkt sich also auf journalistische Inhalte und klammert Universalsuchmaschinen aus.

Die vorliegende Studie möchte zur Erweiterung des Forschungsstandes in diesem Bereich beitragen. Mittels einer nutzerzentrierten Inhaltsanalyse untersucht sie die Qualität der Trefferlisten von fünf Universalsuchmaschinen - Google, Bing, Lycos, Ask und Ixquick - zu sechs gesellschaftlich relevanten, komplexen und kontroversen Themen, bei denen qualitativ hochwertige Suchergebnisse besonders wichtig sind, damit sich die Nutzer umfassend informieren können. Anhand von vier Qualitätskriterien - Benutzerfreundlichkeit, Relevanz, Gattungsvielfalt und Aktualität - prüft sie, ob die Suchmaschinen die Voraussetzungen dafür bieten, dass die Nutzer ihren Rollen als Konsumenten und als Bürger (Hasebrink 2013) gerecht werden können.

\section{Suchmaschinenqualität aus normativer Perspektive - ein Begründungsvorschlag}

Der Begriff Qualität (lat. qualitas) bezeichnet eigentlich wertneutral die Eigenschaften eines Objekts, ist oft aber positiv konnotiert, weil mit Qualität die Erfüllung bestimmter (hoher) Standards assoziiert ist. Was als qualitätsvoll betrachtet wird, ist das Ergebnis eines Bewertungsprozesses, differiert also nach betrachtetem Objekt, Standpunkt des Betrachters und Kontext der Qualitätseinschätzung. Beispielsweise haben Medienunternehmen, Journalisten, Rezipienten und Wissenschaftler unterschiedliche Ansichten darüber, welche Medien(inhalte) qualitativ hochwertig sind. Diese Einschätzung hängt zudem ab von der Mediengattung (z. B. Print, Rundfunk, Online), der Erscheinungshäufigkeit (z. B. täglich, wöchentlich, monatlich), der Funktion der Mediennutzung (z. B. Information, Unterhaltung) und der Zielgruppe des Mediums (Ruß-Mohl 1992, S. 85). Weil Qualität also relational ist und es keine feststehende Begriffsdefinition oder allgemeingültige Kriterien geben kann, erfordern Qualitätsanalysen von Medienprodukten, sich für eine Perspektive auf Qualität zu entscheiden, sie also z. B. aus technischer, ökonomischer, normativer oder nutzerorientierter Sicht zu untersuchen (Arnold 
2009; Hasebrink 2013, S. 4). Es stellt sich die Frage, aus welcher Perspektive die Qualität von Suchmaschinen sinnvoll zu bewerten ist.

Bei einem interaktiven Medium wie Suchmaschinen bietet es sich an, die „Beziehung zwischen Medienangebot und Publikum“ (Mehlis 2014, S. 256) in den Blick zu nehmen, die Qualität also aus der Nutzerperspektive zu betrachten. Hiermit befasst sich die informationswissenschaftliche Suchmaschinenforschung. Neben technischen Kriterien (etwa der Größe, Vollständigkeit und Aktualität des Index, z. B. Deka \& Lahkar 2010) prüft sie vor allem die Fähigkeit der Suchmaschinen zum Auffinden von Informationen oder Dokumenten (Retrievaleffektivität). In sogenannten Retrievaltests bewerten potenzielle Nutzer („Juroren“) hierfür die Relevanz von Suchergebnissen für vorgegebene Suchanfragen. Als Hilfestellung erhalten sie oft Hinweise zum zugrundeliegenden Informationsbedürfnis, das - abhängig von Nutzer und Situation - sehr unterschiedlich aussehen kann (Lewandowski 2015, S. 215). Idealtypisch lassen sich mit Atkin (1973) z. B. drei Informationsbedürfnisse differenzieren: Am einfachsten zu befriedigen ist das konative Orientierungsbedürfnis durch Aktionswissen (z. B. Wettervorhersage, Verkehrsinformationen, Öffnungszeiten). Anspruchsvoller ist die Befriedigung des kognitiven und des affektiven Orientierungsbedürfnisses durch Überblicks- (z. B. Hintergrundwissen über Politik) bzw. Orientierungswissen (z. B. Bewertung politischer Themen). In Retrievaltests schnitt Google bis vor wenigen Jahren besser ab als die Mitbewerber (Deka \& Lahkar 2010; Lewandowski 2008), mittlerweile unterscheidet sich die so gemessene Qualität verschiedener Suchmaschinen aber kaum noch (Lewandowski 2013).

Obwohl Retrievaltests wichtige Ergebnisse zur Qualitätsbewertung durch die Nutzer selbst bezogen auf unterschiedliche Informationsbedürfnisse liefern (Lewandowski 2015, S. 219), reichen sie aus kommunikationswissenschaftlicher Sicht nicht aus, um die Qualität von Suchmaschinen zu beurteilen. Denn durch die Fokussierung auf die Nutzer, denen vor allem das schnelle und bequeme Auffinden der gesuchten Informationen wichtig ist (Stark et al. 2014), vernachlässigen sie die normative Perspektive, welche die kommunikationswissenschaftliche Debatte um Medienqualität entscheidend prägt. ${ }^{2}$ Im Mittelpunkt steht dabei die Frage, ob die Medien hinreichende Voraussetzungen für eine umfassende Meinungs- und Willensbildung bieten. Wichtige medienrechtliche Bezugspunkte sind die öffentliche Aufgabe der Presse und der Auftrag des öffentlich-rechtlichen Rundfunks. Suchmaschinen fehlt zwar eine den traditionellen Massenmedien vergleichbare normative Rückbindung. Mehrere Gründe sprechen aber trotzdem dafür, Qualitätskriterien für journalistische Medien auf sie zu übertragen:

(1) Aus Sicht der Nutzer, insbesondere der jüngeren, hat das Internet als Quelle politischer Information deutlich an Bedeutung gewonnen (van Eimeren 2015). Neben journalistischen Angeboten im Netz ziehen sie dafür zunehmend soziale Netzwerke wie Facebook, Nachrichtenaggregatoren wie Google News und eben auch Google als Suchmaschine heran. Einer repräsentativen Befragung von Hasebrink \& Schmidt (2013, S. 8) zufolge ist Google für die deutsche Bevölkerung die zweitwichtigste Informationsquelle für die politische Meinungsbildung - nach der Tagesschau, aber noch vor der Bild-Zeitung. Obwohl Suchmaschinen selbst „keine eigenen Angebote zur politischen Meinungsbildung “ (ebd.) machen, steuern sie allein durch ihre Vermittlerfunktion beim Zugang zu meinungsrelevanten Themen den Zugang zu Themen aller Art und die gesellschaftliche Wahrnehmung von deren Wichtigkeit (ebd.). Hinzu kommt, dass die Nutzer

2 Das gilt auch für eine medienökonomische Perspektive, die - etwas verkürzt formuliert - dasjenige Angebot für das beste erachtet, das sich am besten „verkauft“, womit sich die Frage nach der besten Suchmaschine eindeutig mit „Google“ beantworten ließe. 
bei journalistischen Angeboten im Netz durchaus Wert auf traditionell-publizistische Qualitätskriterien wie Glaubwürdigkeit, Unabhängigkeit und Themenkompetenz legen (Neuberger 2012, S. 44) - und äquivalent zu solchen nutzen sie Suchmaschinen offenbar. Bei Suchmaschinen sind ihnen ebenfalls normative Kriterien wie Neutralität und Objektivität wichtig (Stark et al. 2014).

(2) Auch Medienpolitik und -recht ziehen zunehmend Parallelen zwischen Suchmaschinen und traditionellen Massenmedien. Der Bundesgerichtshof kommt in seinem "Autocomplete-Urteil“ $2013 \mathrm{zu}$ dem Schluss, dass Suchmaschinen eine dem Journalismus vergleichbare Leistung erbringen, weil insbesondere Google nicht als reiner Informationsvermittler, sondern auch als Informationsanbieter auftritt. Denn indem die Suchmaschine den Zugang zu Inhalten erleichtert oder erschwert, kann sie „theoretisch über die Existenz eines Angebots oder einer Meinung im Netz entscheiden“" (Dörr \& Schuster 2014, S. 263) und beschränkt sich eben nicht auf die rein technische Vermittlung. Und die 2015 eingerichtete Bund-Länder-Kommission zur Medienkonvergenz betont die Rolle von Suchmaschinen für die Meinungsvielfalt, indem sie die Regulierung von Intermediären und speziell Suchmaschinen zu einem ihrer Schwerpunktthemen erklärt (Presse- und Informationsamt der Bundesregierung 2015).

(3) Schließlich lehnt die Selbstkontrolle Suchmaschinen, gegründet 2005 von den bekanntesten Anbietern in Deutschland unter dem Dach der Freiwilligen Selbstkontrolle Multimedia-Diensteanbieter (FSM e. V.), ihren Verhaltenskodex an journalistische Normen an (Selbstkontrolle Suchmaschinen 2004), etwa Transparenz und die Wahrung der Meinungsfreiheit. Sogar die Anbieter selbst orientieren sich demzufolge an normativ begründeten Qualitätskriterien.

\section{Qualität aus Sicht der Mediennutzer als Konsumenten, Bürger und Rechteinhaber}

Um die Qualität von Suchmaschinen umfassender beurteilen zu können, müssen beide Perspektiven - die nutzerorientierte und die normative - miteinander in Einklang gebracht werden. Ein Ansatz von Hasebrink $(2012 ; 2013)$ zur Differenzierung der Nutzerperspektive bietet dazu die Möglichkeit. Ausgangspunkt seiner Überlegungen ist die Beobachtung, dass die Nutzer in der Debatte um Medienqualität bislang nur aus einer Perspektive betrachtet werden: „als Konsumenten von Medienangeboten“ (Hasebrink \& Herzog 2009, S. 14). Basierend auf dem Uses-and-Gratifications-Ansatz wird davon ausgegangen, dass ihre Mediennutzung nur der individuellen Nutzenmaximierung diene, und ihr Qualitätsverständnis wird ausschließlich an der Nutzung oder Nichtnutzung von Angeboten festgemacht. Qualität bemisst sich folglich an der Reichweite - die wiederum ambivalent bewertet wird: Verstanden als Akzeptanz ist sie ein Qualitätsindikator, verstanden als Popularität hingegen ein Indikator mangelnder Qualität (Hasebrink 2013, S. 7). Widersprüche resultieren auch daraus, dass die individuellen Nutzerbedürfnisse häufig mit normativen Kriterien kontrastiert werden, denen sie diametral entgegenzustehen scheinen (Hasebrink \& Herzog 2009, S. 15).

Gerade im Bereich öffentlicher Kommunikation greift die Konzeptionierung des Rezipienten als Konsument allerdings zu kurz (ebd.). „Medienangebote erweisen sich erst dann als qualitätsvoll, wenn Nutzer mit ihnen bestimmte kommunikative Funktionen verwirklichen." (Hasebrink 2013, S. 5) Die Nutzer erwarten von den Medien Unterschiedliches - und in jedem Fall mehr als die Befriedigung ihrer individuellen Bedürfnisse (Neuberger 2012, S. 44). Um dem Rechnung zu tragen, differenziert Hasebrink (2012; 2013) - angelehnt an Nutzermodelle in der Kommunikationspolitik (Webster \& Phalen 1994) - drei Nutzerrollen, in denen verschiedene Bedürfnisse der Nutzer zum 
Ausdruck kommen: die des Konsumenten, des Bürgers und des Rechteinhabers. Diese im Folgenden beschriebene analytische Dreiteilung veranschaulicht die Vielschichtigkeit der Qualitätsbewertung von Medien und lässt sich gut auf die Suchmaschinennutzung übertragen.

(1) In der Konsumentenrolle bestimmen die Gratifikationen, die einzelnen Medien zugeschrieben werden - etwa Information, Unterhaltung und Spannung -, was die Nutzer als qualitativ hochwertig einstufen. Ihr Qualitätsurteil hängt also davon ab, was ihnen an Medienangeboten wichtig ist, und kann abhängig von Person, Situation und Medium stark variieren (Hasebrink 2013, S. 8). Allgemein legen viele Rezipienten Wert darauf, dass Medienangebote einen Mehrwert bieten - sei es im instrumentellen (z. B. zur Information über ein Thema), sozialen (z. B. zur Anschlusskommunikation) oder intrinsischen Sinn (z. B. zur Unterhaltung) (Arnold 2006, S. 425).

(2) Die Qualitätsbewertung aus Sicht der Bürgerrolle orientiert sich an gesellschaftlichen Wertvorstellungen, also an den Merkmalen, die Medienangebote aufweisen sollen, um ihrer gesellschaftlichen Verantwortung nachzukommen, etwa Vielfalt, Ausgewogenheit und Transparenz. In dem Maße, in dem sie diesen gesellschaftlichen Anforderungen nachkommen, ermöglichen sie den Nutzern eine freie, umfassende Meinungsund Willensbildung und befähigen sie zur Wahrnehmung ihrer Bürgerrolle (Hasebrink 2013, S. 8). Entsprechende Qualitätskriterien werden meist aus demokratietheoretischen Überlegungen abgeleitet (z. B. Weßler \& Rinke 2014), wobei die Informations- und Meinungsvielfalt besonders zentral ist.

(3) In der Rolle des Rechteinhabers wird der Nutzer als schutzbedürftiges Individuum betrachtet, das die Möglichkeit haben muss, seine „[a]ls relevant erachtete[n] Schutzbedürfnisse" (Hasebrink 2013, S. 8) zu verteidigen. Diese überschneiden sich stark mit Rechtsnormen, z. B. Persönlichkeitsrechten, sittlichen Empfindungen und der Menschenwürde. Es geht um die Frage, inwiefern Medienangebote diese Schutzbedürfnisse verletzen und welche sozialen Kosten dadurch entstehen (Hasebrink 2012, S. 61).

In der Suchmaschinennutzung findet diese analytische Dreiteilung ihre Entsprechung in der empirischen Realität, wie eine repräsentative Befragung der deutschen Internetnutzer vom Mai 2013 zeigt (Stark et al. 2014; Tabelle 1): Anhand von zwölf Qualitätskriterien wurden die Nutzer gebeten anzugeben, was für sie persönlich eine gute Suchmaschine ausmacht. Diese Kriterien lassen sich mittels einer Faktorenanalyse (Hauptkomponentenanalyse) ${ }^{3}$ auf drei Dimensionen reduzieren, die die drei Nutzerrollen ziemlich genau widerspiegeln.

(1) Die Konsumentenrolle vereint die schnelle Anzeige von Ergebnissen, die Anzeige relevanter Ergebnisse und eine übersichtliche, unkompliziert bedienbare Startseite. Darin kommt der vordringliche Wunsch der Nutzer zum Ausdruck, mittels Suchmaschinen einfach, schnell und unkompliziert zu finden, wonach sie suchen. Genau diese Kriterien erfahren in der Einzelbetrachtung die höchste Zustimmung, was die Ergebnisse von Machill et al. (2003) und Bar-Ilan et al. (2007) zur Suchmaschinenqualität aus Nutzersicht bestätigt. ${ }^{4}$ Die Konsumentenrolle scheint also in aller Regel zu dominieren, worauf auch

3 Die Zahl der Faktoren wurde anhand dreier Kriterien bestimmt: dem Kaiser-Kriterium (Eigenwert > 1), dem Ellenbogenkriterium (Scree-Plot) und möglichst ausgewogenen Kommunalitäten. Die drei Dimensionen erklären zusammen 59 \% der Varianz.

4 Einige weitere Studien zur Nutzersicht auf Suchmaschinen verweisen auf die Bedeutung der wahrgenommenen Qualität der Suchergebnisse für die Bewertung der Suchmaschinen, differenzieren aber nicht, was sie unter Qualität verstehen (z. B. Purcell et al. 2012) oder geben die verwendeten Items nicht an (z. B. Veloutsou \& McAlonan 2012), weshalb sich ihre Befunde schwer mit den vorliegenden vergleichen lassen. 


\section{Tabelle 1: Suchmaschinennutzer als Konsumenten, Bürger und Rechteinhaber}

\begin{tabular}{|c|c|c|c|c|}
\hline & $\begin{array}{c}\text { äußerst/ } \\
\text { sehr } \\
\text { wichtig } \\
(\%)\end{array}$ & $\begin{array}{l}\text { Konsu- } \\
\text { ment }\end{array}$ & Bürger & $\begin{array}{l}\text { Rechte- } \\
\text { inhaber }\end{array}$ \\
\hline Liefert schnell die Informationen, die man braucht. & 85 & 807 &, 162 &, 162 \\
\hline $\begin{array}{l}\text { Liefert Ergebnisse, die für die Suchanfrage relevant } \\
\text { sind. }\end{array}$ & 83 & ,784 & ,219 &, 080 \\
\hline $\begin{array}{l}\text { Hat eine übersichtliche Startseite, ist einfach und un- } \\
\text { kompliziert zu nutzen. }\end{array}$ & 81 &, 742 & ,166 &, 269 \\
\hline $\begin{array}{l}\text { Gibt Auskunft darüber, wie die Anordnung der Er- } \\
\text { gebnisse zustande kommt. }\end{array}$ & 39 &,- 141 & ,782 &, 135 \\
\hline $\begin{array}{l}\text { Liefert Suchergebnisse, die Anfragen aus unter- } \\
\text { schiedlichen Perspektiven beleuchten. }\end{array}$ & 54 & ,236 & ,742 & ,098 \\
\hline $\begin{array}{l}\text { Fördert interessante und überraschende Informatio- } \\
\text { nen zutage. }\end{array}$ & 59 &, 249 & ,682 &, 111 \\
\hline $\begin{array}{l}\text { Liefert Suchergebnisse von mehreren verschiedenen } \\
\text { Anbietern. }\end{array}$ & 64 & ,475 &, 535 &, 005 \\
\hline $\begin{array}{l}\text { Macht passende Vorschläge für mögliche Suchbegrif- } \\
\text { fe, schon während man seine Anfrage eintippt. }\end{array}$ & 59 & ,199 &, 534 &, 250 \\
\hline $\begin{array}{l}\text { Liefert Suchergebnisse, die nicht durch kommerzielle } \\
\text { Interessen beeinflusst werden. }\end{array}$ & 63 & ,289 &, 506 &, 166 \\
\hline $\begin{array}{l}\text { Achtet darauf, dass nichts angezeigt wird, das Kin- } \\
\text { dern und Jugendlichen schaden könnte, z. B. Gewalt } \\
\text { oder Kinderpornografie. }\end{array}$ & 67 &,- 018 &, 183 & 835 \\
\hline $\begin{array}{l}\text { Warnt, wenn Ergebnisse von nicht vertrauenswürdi- } \\
\text { gen Seiten stammen. }\end{array}$ & 77 &, 283 &, 179 &, 754 \\
\hline Geht sorgsam mit meinen persönlichen Daten um. & 85 &, 461 &, 121 &, 579 \\
\hline Erklärte Varianz (rotiert) & & $22 \%$ & $22 \%$ & $15 \%$ \\
\hline
\end{tabular}

Hauptkomponentenanalyse mit Varimax-Rotation. $\mathrm{KMO}=.885$; Bartlett's Test auf Sphärizität: $\chi^{2}$ $(66)=3709,3 ; \mathrm{p}<.001$. Quelle: eigene Daten. Basis: Repräsentative Stichprobe von 1.012 Internetnutzern.

Frage: Zunächst möchten wir wissen, was für Sie persönlich ganz allgemein eine gute Suchmaschine ausmacht? Wie wichtig sind Ihnen folgende Punkte? Bitte bewerten Sie deren Wichtigkeit auf einer Skala von 1 bis 5 , wobei der Wert 1 „sehr wichtig“ und der Wert 5 „völlig unwichtig“ bedeutet. Mit den Werten dazwischen können Sie Ihre Bewertung abstufen.

die übliche Nutzungsweise von Suchmaschinen hindeutet: Die Rezipienten sind nicht bereit, hohen kognitiven Aufwand in die Suche zu investieren, lassen sich heuristisch von bisherigen Erfahrungswerten und oberflächlichen Hinweisreizen leiten und beenden ihre Suche meist auf der ersten Ergebnisseite, häufig sogar bei den ersten ein bis drei Treffern (z. B. Stark et al. 2014; Wirth et al. 2007).

(2) Der Bürgerrolle werden fünf normativ begründete Qualitätskriterien zugeordnet: die Transparenz über die Kriterien, nach denen die Ergebnisse angeordnet sind; die Vielfalt von Anbietern und Perspektiven, die Freiheit von kommerziellen Interessen und 
die Anzeige interessanter und überraschender Ergebnisse. Nicht in dieses Muster passt lediglich die Autocomplete-Funktion - Suchvorschläge, welche die Suchmaschine während des Eintippens der Anfrage macht -, die inhaltlich der Konsumentenrolle näher steht.

(3) Die Rolle des Rechteinhabers bündelt die Sorgen um Jugend- und Datenschutz sowie um die Vertrauenswürdigkeit der angezeigten Inhalte.

\section{Kriterien zur Messung der Qualität von Trefferlisten}

Die vorliegende Studie prüft mittels einer nutzerzentrierten Inhaltsanalyse, inwieweit die Trefferlisten verschiedener Suchmaschinen die Voraussetzungen dafür bieten, dass die Nutzer die Konsumenten- und die Bürgerrolle einnehmen können. Qualitätsmängel aus Sicht des Rechteinhabers lassen sich so hingegen kaum untersuchen. Einige davon sind zwar inhaltsanalytisch messbar, dürften aufgrund ihrer engen Verwandtschaft mit Rechtsnormen aber kaum auf den ersten Ergebnisseiten auftreten. Über andere Kriterien wie die Einhaltung des Datenschutzes können Trefferlisten keine Auskunft geben. Deshalb fokussiert die übergeordnete Forschungsfrage F1 auf die beiden anderen Rollen: Wie gut genügen die Trefferlisten von Suchmaschinen den Qualitätsansprüchen der Nutzer in ibrer Konsumenten- und Bürgerrolle?

Angelehnt an die Befragungsergebnisse (Tabelle 1) sowie die Befunde von Machill et al. (2003) werden vier Kriterien betrachtet: In der Konsumentenrolle legen die Nutzer vor allem Wert auf die (1) Benutzerfreundlichkeit der Suchmaschinen und die (2) Relevanz der Suchergebnisse. Zwei zentrale Voraussetzungen dafür, dass die Nutzer ihre Bürgerrolle erfüllen können, sind die (3) Gattungsvielfalt und (4) Aktualität der Suchergebnisse. Hierbei handelt es sich um Kriterien, die sich auf Basis der Informationen in Trefferlisten gut untersuchen lassen.

\subsection{Benutzerfreundlichkeit}

Die Benutzerfreundlichkeit - nach Quirmbach (2012, S. 60) auch Usability oder Gebrauchstauglichkeit - ist ein onlinespezifisches Kriterium, das sowohl in der informations- als auch in der kommunikationswissenschaftlichen Qualitätsforschung eine Rolle spielt. Benutzerfreundlich sind Onlinemedien, wenn ihre Benutzerführung drei Leitkriterien folgt: Effektivität (wie einfach finden die Nutzer die gewünschten Informationen?), Effizienz (wie schnell gelangen sie ans Ziel?) und Zufriedenheit (wie zufrieden sind sie mit den Ergebnissen?) (ebd.). Im Fall von Suchmaschinen bedeutet das, möglichst schnell die gewünschten Informationen zu erhalten, entsprechend der vordringlichen Erwartungen der Nutzer (Tabelle 1). Die benutzerfreundliche Gestaltung einer Suchmaschine trägt kurzfristig zum Sucherfolg der Nutzer und langfristig dazu bei, dass sie dauerhaft genutzt wird. Hilfreich dafür ist beispielsweise eine Startseite, die so strukturiert ist, dass die Nutzer jederzeit die Übersicht darüber behalten, wo sie sich gerade befinden. Da sich die Nutzer stark an fettgedruckten Suchbegriffen in Überschriften und Teasern als „heuristic cues“ orientieren (Stark et al. 2014), dürfte diese Hervorhebung die Orientierung und damit die Benutzerfreundlichkeit steigern. Auch die Autocomplete-Funktion (Stark et al. 2014) und Suchvorschläge in Form von „verwandten Suchanfragen“ (Google 2013) können dazu beitragen. Letztere sind aber nur vorteilhaft, wenn sie inhaltlich zur Suchanfrage passen und nicht in zu großer Zahl angezeigt werden, wodurch die Trefferlisten unübersichtlich würden.

Das gilt auch für Werbung, über die sich die meisten Suchmaschinen fast ausschließlich finanzieren. Einnahmen akquirieren sie erst, wenn die Nutzer die Anzeigen auf den 
Trefferlisten anklicken, weshalb es lukrativ ist, diese möglichst nah bei den Suchergebnissen zu platzieren (Neuberger 2011, S. 107). Zu viele Anzeigen sind der Benutzerfreundlichkeit aber abträglich, weil sie von den Suchergebnissen ablenken und die Differenz zwischen ihnen und den organischen Treffern nicht allen Nutzern bewusst ist. Werden Werbetreffer erkannt, empfinden viele Nutzer sie als lästig (Stark et al. 2014), selbst wenn auch sie in manchen Fällen für die Suche relevant sein können (z. B. bei Produktsuchen). Aus diesen Überlegungen leitet sich Forschungsfrage F1a ab: Wie benutzerfreundlich sind die Trefferlisten der Suchmaschinen?

\subsection{Relevanz}

Relevanz $z^{5}$ meint die Bedeutsamkeit einzelner Informationen, die es in der Unübersichtlichkeit der Informationsflut zu identifizieren gilt. Sie dient der Reduktion von Komplexität, einer zentralen Funktion von Journalismus (Hagen 1995, S. 71) und Suchmaschinen (Stark et al. 2014) gleichermaßen. In den traditionellen Massenmedien nehmen die Journalisten Relevanzzuschreibungen vor und orientieren sich dabei z. B. an den Nachrichtenfaktoren. Bei Suchmaschinen filtert ein Algorithmus, basierend auf technischen Kriterien, die am besten zur Suchanfrage passenden Inhalte heraus (Latzer et al. 2014, S. 6). Will man prüfen, ob daraus relevante Suchergebnisse resultieren, stellt sich dasselbe Problem wie bei der Untersuchung der Qualität von Suchmaschinen allgemein: Auch Relevanz ist nicht absolut bestimmbar, sondern hängt ab von Betrachter und Kontext (Bar-Ilan et al. 2007, S. 1264). Zu fragen ist also nicht, ob eine Information per se relevant ist, sondern für wen und in welcher Situation.

Je nach der Rolle, welche die Nutzer gerade einnehmen, werden sie Unterschiedliches als relevant einstufen: In der Konsumentenrolle erwarten sie von Suchmaschinen relevante Informationen im Sinne von „passend zur Suchanfrage“. In der Bürgerrolle hingegen sind Informationen relevant, die zur Meinungsbildung beitragen. Dass die durch Algorithmen generierten Trefferlisten Relevanzzuschreibungen in diesem zweiten Sinn leisten können, zweifeln Beiler (2013, S. 381) und Bar-Ilan et al. (2007, S. 1264) aufgrund ihrer Ergebnisse an. Allein auf Basis der Trefferlisten wie in der vorliegenden Studie lässt sich dies jedoch kaum prüfen. Dazu müssten auch die Websites, auf die verlinkt wird, analysiert werden. Forschungsfrage F1b beschränkt sich daher auf die Relevanz aus Konsumentensicht: Wie relevant sind die Trefferlisten der Suchmaschinen, gemessen an ibrer Passung zur Suchanfrage?

\subsection{Gattungsvielfalt}

Vielfalt - ein zentrales Qualitätskriterium, das hier der Bürgerrolle zugeordnet wird meint die Verschiedenartigkeit des Medienangebots: Die Medien sollen ein möglichst breites Spektrum an Informationen und Positionen bereitstellen (Schatz \& Schulz 1992). Diese Vielfalt der Meinungen ist laut Mill (1956, S. 58) notwendige Voraussetzung der Wahrheitsfindung. Sie bildet - beruft man sich auf die durch Mills Ideen geprägte Metapher des "marketplace of ideas" - die Voraussetzung für einen gesellschaftlichen und rationalen Diskurs, der nach dem Idealbild der Demokratietheorie zu einer differenzierten Meinungsbildung führt (Zerback 2013). Doch gerade die Vielfalt stellen verschiedene Autoren für Suchmaschinen und insbesondere Google in Frage (Dörr \&

5 Für die vorliegende Studie ist die kommunikationswissenschaftliche Sichtweise auf Relevanz von Belang. Zur informationswissenschaftlichen Diskussion des Relevanzbegriffs siehe Mizzarro (1997) und Saracevic (2007). 
Schuster 2014; Pariser 2011). Zwar generieren die meisten Suchmaschinen eine enorme Trefferzahl, stellen damit den Zugang zu einer schier unbegrenzten Informationsvielfalt her.

Gerade darin liegt aber zugleich das Problem: Wirkliche Vielfalt ist nur gegeben, wenn die Nutzer davon Gebrauch machen (Dörr \& Schuster 2014) - und das tun sie mitnichten, sondern nehmen nur die weit vorne positionierten Treffer, vertrauen also der Relevanzzuschreibung der Suchmaschine (Bar-Ilan et al. 2007, S. 1264). Damit der Nutzer „wirkliche Selektionsalternativen hat und aus einer größtmöglichen Vielfalt auswählen" (Beiler 2013, S. 100), also seine Bürgerrolle wahrnehmen kann, müssen die tatsächlich wahrgenommenen Treffer vielfältig sein. Das mag beim „Googeln“ nach Aktionswissen - simplen Fakten wie Öffnungszeiten oder Verkehrsverbindungen - keine große Rolle spielen. Bei der Suche nach Überblicks- und Orientierungswissen (Atkin 1973) wie in der vorliegenden Studie ist es aber entscheidend.

Die kommunikationswissenschaftliche Qualitätsforschung unterscheidet zwischen inhaltlicher und struktureller Vielfalt (Schatz \& Schulz 1992). Die inhaltliche Vielfalt, die sich z. B. auf die Repräsentation verschiedener Lebensbereiche, Perspektiven und Meinungen bezieht, lässt sich allein auf Basis von Trefferlisten zwar nicht untersuchen - wohl aber die strukturelle Vielfalt, die Repräsentation unterschiedlicher Sparten oder Genres. Übertragen auf Suchmaschinen lässt sie sich daran messen, ob die Treffer von verschiedenartigen Anbietern stammen, also verschiedenen Gattungen zuzurechnen sind - das Qualitätskriterium aus Bürgersicht, das den Nutzern selbst laut Tabelle 1 am wichtigsten ist. Verschiedene Treffergattungen bereiten Informationen unterschiedlich auf, und dadurch steigt die Wahrscheinlichkeit, dass die Nutzer mit Informationen aus verschiedenen Blickwinkeln und alternativen Sichtweisen konfrontiert werden, etwa in Blogs oder Foren. Ob die Voraussetzungen dafür gegeben sind, untersucht Forschungsfrage F1c: Wie groß ist die Gattungsvielfalt der Trefferlisten?

\subsection{Aktualität}

Die Aktualität im Sinne (annähernder) Gleichzeitigkeit unterscheidet die Informationsleistung von Nachrichten „von der anderer primär wissensvermittelnder Diskurstypen (Geschichte, Wissenschaft...)“ (Hagen 1995, S. 128) und ist somit ein zentrales Wesensmerkmal des Journalismus. Sowohl Bürger als auch gesellschaftliche Entscheider brauchen aktuelle Informationen, um über den gegenwärtigen Stand der Dinge informiert zu sein und sachgerechte Entscheidungen treffen zu können, weshalb die Aktualität der Bürgerrolle zugeordnet wird. Bei Onlinemedien wächst der Stellenwert der Aktualität enorm, weil deren Inhalte rund um die Uhr aktualisiert werden können und im Kampf um die Aufmerksamkeit der Rezipienten sogar müssen.

Suchmaschinen dienen unter anderem der Suche nach Nachrichten, womit Aktualität auch für sie zu einem Qualitätskriterium wird. Die Nutzer erwarten aktuelle Ergebnisse von einer guten Suchmaschine (Machill et al. 2005, S. 129) und ziehen als Indikator dafür die angezeigten Veröffentlichungsdaten der Treffer heran (Stark et al. 2014). ${ }^{6}$ Wie gut die Suchmaschinen diesem Kriterium gerecht werden, prüft Forschungsfrage F1d: Wie aktuell sind die Trefferlisten der Suchmaschinen?

6 Problematisch ist, dass die Aktualität der Treffer manipuliert werden kann, indem die zugrunde liegenden Websites permanent aktualisiert werden, s. Beiler (2013, S. 110). 


\section{Methode}

Um die Forschungsfragen zu beantworten, werden die Trefferlisten von fünf Suchmaschinen inhaltsanalytisch untersucht: Google (96\% Marktanteil), Bing (1,4\%) und Ask $(0,8 \%)$ waren im Untersuchungsjahr die meistgenutzten Suchmaschinen in Deutschland (ComScore 2013, S. 43). ${ }^{7}$ Die beiden anderen Anbieter sollen bestimmte Typen von Suchmaschinen repräsentieren: $L y \cos$ steht für einst große Anbieter, die mittlerweile von Google verdrängt wurden (O'Neill 2013), Ixquick - eine Meta-Suchmaschine, die keine persönlichen Daten speichert (ixquick.com 2013) - für alternative Suchmaschinen, die anderen Geschäftsprinzipien folgen als Google.

Dem Ansatz der nutzerzentrierten Inhaltsanalyse nach Rössler und Wirth (2001) folgend, beschränkt sich die Analyse ebenso wie die meisten Rezipienten auf die jeweils erste Trefferlistenseite - also das, was die Rezipienten vor sich sehen, wenn sie ihre Selektionsentscheidung treffen. Um die Trefferlisten zu generieren, wurden sechs Suchanfragen zu gesellschaftlich relevanten, kontroversen Themen durchgeführt. Diese betreffen verschiedene Gesellschaftsbereiche und sind dem Überblicks- und Orientierungswissen (Atkin 1973) zuzuordnen, wofür hochwertige Trefferlisten besonders wichtig sind. Damit die Trefferlisten nicht ausschließlich tagesaktuelle Massenmedien beinhalten, was die Varianz der Treffer (insbesondere hinsichtlich Gattungsvielfalt und Aktualität) von vornherein einschränken würde, fiel die Entscheidung auf sechs gesellschaftlich relevante „Dauerbrennerthemen“. Ausgehend vom typischen Nutzerverhalten - Suchanfragen in Deutschland bestehen aus durchschnittlich 1,66 Wörtern (Höchstötter \& Koch 2009) - wurden Suchanfragen aus ein bis zwei für das jeweilige Thema zentralen Begriffen formuliert: „Stress Schulkinder“, „Rundfunkbeitrag “", „Organspende “, „Mindestlohn“, „Homo-Ehe“, „Videoüberwachung Deutschland“.8

Zwecks größtmöglicher Vergleichbarkeit der Trefferlisten wurden zunächst alle Cookies gelöscht, Adblocker deaktiviert und bei Google die Personalisierung ausgeschaltet. Anschließend wurden alle Suchanfragen mit sämtlichen Suchmaschinen vom selben Computer aus zeitgleich am 9. Juli 2013 ausgeführt und die Trefferlisten mit Hilfe des Plug-in „ScrapBook“ lokal gespeichert. Die Analyse basiert auf 30 Trefferlisten (je sechs Listen von den fünf verschiedenen Suchmaschinen), die in der Regel je zehn Treffer ${ }^{9}$ enthielten; nur in wenigen Fällen wich die Trefferzahl geringfügig davon ab, sodass insgesamt 312 Treffer analysiert wurden (Google: 66, Bing: 66, Ask: 60, Lycos: 60, Ixquick: 60). Die im Folgenden beschriebenen Qualitätsindikatoren werden entweder auf Ebene der gesamten Trefferlisten (Listenebene) oder auf Ebene der einzelnen Treffer (Trefferebene) untersucht. Die auf den Trefferlisten verlinkten Websites werden nicht analysiert, denn das Ziel der Untersuchung ist, die Trefferlisten zu bewerten, welche die Nutzer vor sich sehen und aus denen sie ihre Auswahl treffen. Der Wertebereich aller Indikatoren reicht von 0 für die kleinst- bis 1 für die größtmögliche Qualität.

(1) Die Benutzerfreundlichkeit wird mittels drei Indikatoren gemessen: (a) Auf der Trefferebene wird der Anteil der fettgedruckten an allen Suchwörtern in Titel und

7 Yaboo und T-Online, die meistgenutzten Suchmaschinen in Deutschland nach Google und Bing, werden nicht untersucht, weil beide keine eigenständigen Suchmaschinen sind: T-Online nutzt für seine Trefferlisten Google, Yahoo verwendet Bing.

$8 \mathrm{Zu}$ vier Themen wurden sowohl einfache als auch komplexe Anfragen formuliert. Dies veränderte die Ergebnisse aber nur marginal, weshalb sich die Darstellung auf die einfachen Suchanfragen beschränkt.

9 Auch Retrievaltests basieren meist auf den ersten zehn Treffern pro Suchanfrage (Lewandowski 2015, S. 220). 
Teasertext (in Prozent) bestimmt. Auch sich wiederholende fettgedruckte Suchwörter werden als nutzerfreundlich gewertet, weil sie die Informationsverarbeitung der Nutzer beschleunigen. (b) Die Werbefreiheit basiert auf der Überlegung, dass die Nutzer umso leichter zu den gewünschten Ergebnissen gelangen, je weniger Werbung die Trefferliste enthält. Dazu wird zunächst auf der Listenebene die Anzahl der Werbeanzeigen pro Treffer bestimmt. Der Grad der Werbefreiheit wird berechnet, indem der Werbeanteil jeder Liste durch den insgesamt höchsten Werbeanteil dividiert und - weil ein geringer Werbeanteil als qualitativ hochwertig gilt - von 1 subtrahiert wird. Ein Wert von 0 steht dabei für die insgesamt in den Daten vorkommende höchste Anzahl an Werbeanzeigen pro Treffer, also die im Verhältnis geringste Werbefreiheit, ein Wert von 1 für das Fehlen von Werbeanzeigen, also die größtmögliche Werbefreiheit. (c) Ebenfalls auf der Listenebene wird untersucht, ob die Suchmaschinen den Nutzern ergänzend zur Trefferliste Suchvorschläge machen und, falls ja, ob diese inhaltlich zur Suchanfrage passen. Als inhaltlich passend $(=1)$ werden Suchvorschläge codiert, die gemäß Suchszenario (siehe unten) zumindest grob relevant für die Suchanfrage sind. Darauf basierend wird der Anteil der inhaltlich passenden an allen Suchvorschlägen errechnet. Negativ gewertet werden nicht passende, doppelte oder gänzlich fehlende Suchvorschläge $(=0)$. Um die Benutzerfreundlichkeit der Suchmaschinen insgesamt bewerten zu können, wird als Index das arithmetische Mittel aus diesen drei Indikatoren berechnet.

(2) Hinsichtlich der Relevanz ist von Interesse, (a) wie viele der eingegebenen Suchwörter mindestens einmal im Titel und/oder Teasertext des Suchtreffers vorkommen und (b) wie viele davon in einem logischen Zusammenhang miteinander stehen (beides in Prozent), wobei auch Synonyme berücksichtigt werden (z. B. „Schulkind“ oder „Schülerin“ beim Suchwort „Schüler“). Im Fall von Einwort-Suchanfragen wird ein logischer Zusammenhang als immer vorhanden angesehen, sofern der Treffer das Suchwort enthält (=1). Fehlt das Suchwort, wird 0 codiert. (c) Zudem wird geprüft, inwiefern die Treffer inhaltlich zur Suchanfrage passen. Da diese Einschätzung vom Betrachter abhängt, wurde als Codierhilfe - orientiert an den Informationen, die Juroren in Retrievaltests erhalten (Lewandowski 2015, S. 219) - für jede Suchanfrage in einem Suchszenario das dahinterstehende Informationsbedürfnis beschrieben und skizziert, welche Treffer wie gut geeignet sind, um es zu befriedigen. ${ }^{10}$ Die Treffer wurden auf einer dreistufigen Skala von "nicht relevant“ $(=0)$ über „teilweise relevant“ $(=0.5)$ bis zu „relevant“ (=1) eingestuft. Auch für die Gesamtbewertung der Relevanz wird als Index das arithmetische Mittel der drei zugrunde liegenden Indikatoren berechnet.

(3) Zur Messung der Gattungsvielfalt wird jeder Treffer einer Gattung zugewiesen (z. B. Internetableger eines klassischen Massenmediums, Wiki, Blog) und auf der Listenebene das Maß der relativen Entropie als Vielfaltsindex mit einem Wertebereich von $O$ (= kleinstmögliche Vielfalt, alle Treffer lassen sich einer einzelnen Gattung zuordnen) bis 1 (= größtmögliche Vielfalt, gleichmäßige Verteilung der Treffer auf alle Gattungen) berechnet (Franzmann \& Wagner 1999, S. 78).

10 Beispiel: Szenario zum Thema Mindestlohn: Der Nutzer möchte sich über verschiedene Regelungen (z. B. Höhe des Mindestlohns in verschiedenen Ländern und Branchen, Gesetze) sowie Facetten der Mindestlohn-Debatte (z. B. Stellungnahmen, Pro/Kontra-Argumente) informieren, um in seinem Umfeld darüber diskutieren zu können. Dazu geeignete Informationen sind relevant (=1). Informationen wie z. B. Seiten von Mindestlohn-Initiativen in der Schweiz sind teilweise relevant, weil sie in einem tiefgreifenden Gespräch unter Umständen nützlich sein können, aber nicht zwangsläufig müssen $(=0,5)$. Übersetzungen und Informationen zur Schreibweise von „Mindestlohn“ werden als nicht relevant eingestuft $(=0)$. 
(4) Die Aktualität wird auf der Trefferebene bestimmt. Dazu werden die angegebenen Daten klassifiziert in Treffer mit Datumsangabe vom Tag der Suchanfrage (=1.0), aus der Woche der Suchanfrage (=0.75), dem Monat oder Vormonat der Suchanfrage (=0.5), zwei Monate vor der Suchanfrage und älter $(=0.25)$ oder ohne Datumsangabe $(=0)$. Das arithmetische Mittel dieses Indikators dient als Aktualitätsindex.

Um die Gesamtqualität der Suchmaschinen vergleichen und Aussagen darüber treffen zu können, wie nahe sie der höchstmöglichen Qualität kommen, werden die Indizes für die vier Kriterien in einem Gesamtindex zusammengefasst. Dazu wird das arithmetische Mittel aus den Indexwerten der Benutzerfreundlichkeit, Vielfalt und Aktualität gebildet und mit dem Indexwert der Relevanz multipliziert. Im Gesamtindex wird die Relevanz also höher gewichtet als die drei anderen Kriterien, ausgehend vom Befund, dass die Nutzer von Suchmaschinen vor allem relevante Ergebnisse erwarten (Tabelle 1). Durch die Multiplikation erhalten Suchmaschinen, die irrelevante Ergebnisse liefern, stets einen niedrigen Indexwert, unabhängig davon, wie sie bei den drei anderen Kriterien abschneiden.

Die Codierung fand im Juni (Pretest) und Juli (Hauptuntersuchung) 2013 statt. Die Inter-Coder-Reliabilität zwischen den zehn Codierern ${ }^{11}$ ist für fast alle Variablen zufriedenstellend (Krippendorff's $\alpha$ : .76 bis 1.0). Lediglich der Koeffizient für die schwer zu codierende inhaltliche Relevanz ist mit .62 niedriger, aber noch akzeptabel.

\section{Ergebnisse}

\subsection{Benutzerfreundlichkeit}

Vergleicht man die Benutzerfreundlichkeit der Suchmaschinen anhand der fettgedruckten Suchbegriffe, sind Tabelle 2 zufolge Google, Ixquick, und Bing gleich benutzerfreundlich. Auffallend ist, dass sie nur die Wörter fett darstellen, die exakt dem eingegebenen Suchbegriff entsprechen. Würden auch Synonyme hervorgehoben, könnte die Benutzerfreundlichkeit noch weiter steigen. Wenig benutzerfreundlich sind in dieser Hinsicht hingegen Lycos und Ask, die gänzlich auf den Fettdruck von Suchbegriffen verzichten und sich damit signifikant von den drei Mitbewerbern unterscheiden.

Dem Ideal der Werbefreiheit kommt eindeutig Google mit durchschnittlich nur einer Anzeige pro zehn Treffer am nächsten und ist daran gemessen benutzerfreundlicher als Lycos und signifikant benutzerfreundlicher als Bing, Ixquick und Ask. Lycos und Bing zeigen 0,3, Ixquick 0,6 und Ask 0,8 Anzeigen pro Treffer an. Dieser Befund erscheint kontraintuitiv angesichts des häufigen Vorwurfs, dass der Google-Konzern stark durch kommerzielle Interessen getrieben sei, wird aber erklärbar vor dem Hintergrund, dass Google durch die Sammlung von Nutzer- und Nutzungsdaten die Anzeigen viel zielgerichteter platzieren kann als die Konkurrenz.

Der dritte Indikator für Benutzerfreundlichkeit ist der Anteil der inhaltlich passenden Suchvorschläge, die ergänzend zur Trefferliste angezeigt werden und die Nutzer bei ihrer Suche unterstützen. Dabei schneidet Google signifikant besser ab als sämtliche Mitbewerber. Die Suchvorschläge von Ask und Bing helfen bei der Beantwortung der Suchanfrage weniger weiter. Lycos und Ixquick bieten keine Suchvorschläge an, sind in diesem Punkt also am wenigsten benutzerfreundlich.

11 An dieser Stelle möchten wir uns bei Carolin Crockett, Jonas Cußler, Saskia Grössl, Sarah Korst und Tatsiana Kuzmenka bedanken, die uns bei der Entwicklung des Codebuchs sowie bei der Durchführung der Codierung unterstützt haben. 
Fasst man die Ergebnisse der drei Indikatoren zur Benutzerfreundlichkeit zu einem Index zusammen (Tabelle 2), lässt sich F1a wie folgt beantworten: Google ist insgesamt die benutzerfreundlichste Suchmaschine und unterscheidet sich darin signifikant von den Konkurrenten. Der Abstand von Lycos erklärt sich dadurch, dass diese Suchmaschine zwei der drei untersuchten Funktionen gar nicht anbietet. Ixquick und Ask hingegen schneiden vergleichsweise schlecht ab, obwohl jeweils zwei der drei Funktionen vorhanden sind. Bing schneidet schlechter ab als Google, obwohl es alle untersuchten Funktionen anbietet, aber keine guten Suchvorschläge macht.

\section{Tabelle 2: Benutzerfreundlichkeit der Trefferlisten}

\begin{tabular}{|c|c|c|c|c|c|c|}
\hline & $\begin{array}{l}\text { Google } \\
\text { (A) }\end{array}$ & $\begin{array}{l}\text { Bing } \\
\text { (B) }\end{array}$ & $\begin{array}{l}\text { Ixquick } \\
\text { (C) }\end{array}$ & $\begin{array}{l}\text { Ask } \\
\text { (D) }\end{array}$ & $\begin{array}{l}\text { Lycos } \\
(\mathrm{E})\end{array}$ & Gesamt \\
\hline $\begin{array}{l}\text { Anteil fettgedruckter } \\
\text { Suchwörter }\end{array}$ & $\begin{array}{c}.81^{\mathrm{D}, \mathrm{E}} \\
(\mathrm{s}=.27) \\
(\mathrm{n}=66) \\
\end{array}$ & $\begin{array}{l}.80 \mathrm{D}, \mathrm{E} \\
(\mathrm{s}=.26) \\
(\mathrm{n}=66)\end{array}$ & $\begin{array}{l}.81^{\mathrm{D}, \mathrm{E}} \\
(\mathrm{s}=.26) \\
(\mathrm{n}=60) \\
\end{array}$ & $\begin{array}{c}.00 \\
(s=.00) \\
(\mathrm{n}=60) \\
\end{array}$ & $\begin{array}{c}.00 \\
(s=.00) \\
(n=60)\end{array}$ & $\begin{array}{c}.50 \\
(s=.44) \\
(n=312)\end{array}$ \\
\hline Werbefreiheit (Index) & $\begin{array}{l}.86^{B, C, D} \\
(s=.21) \\
(n=66)\end{array}$ & $\begin{array}{l}.68^{\mathrm{C}, \mathrm{D}} \\
(\mathrm{s}=.32) \\
(\mathrm{n}=66)\end{array}$ & $\begin{array}{c}.38^{\mathrm{D}} \\
(\mathrm{s}=.19) \\
(\mathrm{n}=60)\end{array}$ & $\begin{array}{c}.22 \\
(s=.27) \\
(n=60)\end{array}$ & $\begin{array}{l}.75 \mathrm{C}, \mathrm{D} \\
(\mathrm{s}=.27) \\
(\mathrm{n}=60)\end{array}$ & $\begin{array}{c}.59 \\
(s=.35) \\
(n=312)\end{array}$ \\
\hline $\begin{array}{l}\text { Anteil der relevanten } \\
\text { Suchvorschläge }\end{array}$ & $\begin{array}{c}.80^{B}, C, D, E \\
(s=.35) \\
(n=66)\end{array}$ & $\begin{array}{c}.31 \mathrm{C}, \mathrm{E} \\
(\mathrm{s}=.27) \\
(\mathrm{n}=66)\end{array}$ & $\begin{array}{c}.00 \\
(s=.00) \\
(n=60)\end{array}$ & $\begin{array}{l}.46^{\mathrm{B}, \mathrm{C}, \mathrm{E}} \\
(\mathrm{s}=.21) \\
(\mathrm{n}=60)\end{array}$ & $\begin{array}{c}.00 \\
(s=.00) \\
(n=60)\end{array}$ & $\begin{array}{c}.32 \\
(s=.38) \\
(n=312)\end{array}$ \\
\hline $\begin{array}{l}\text { Index Benutzer- } \\
\text { freundlichkeit } \\
\text { (MW) }\end{array}$ & $\begin{array}{c}.83^{\mathrm{B}, \mathrm{C}, \mathrm{D}, \mathrm{E}} \\
(\mathrm{s}=.24) \\
(\mathrm{n}=66)\end{array}$ & $\begin{array}{l}.60 \mathrm{C}, \mathrm{D}, \mathrm{E} \\
(\mathrm{s}=.16) \\
(\mathrm{n}=66)\end{array}$ & $\begin{array}{l}.40 \mathrm{D}, \mathrm{E} \\
(\mathrm{s}=.10) \\
(\mathrm{n}=60)\end{array}$ & $\begin{array}{c}.23 \\
(\mathrm{~s}=.13) \\
(\mathrm{n}=60)\end{array}$ & $\begin{array}{c}.25 \\
(\mathrm{~s}=.09) \\
(\mathrm{n}=60)\end{array}$ & $\begin{array}{c}.47 \\
(s=.28) \\
(n=312)\end{array}$ \\
\hline
\end{tabular}

Aa Die Buchstaben hinter den Werten zeigen an, von welchen anderen Werten in derselben Zeile sich der betreffende Wert signifikant unterscheidet (Kleinbuchstaben: $\mathrm{p}<0,05$; Großbuchstaben: $\mathrm{p}<0,01)$.

\subsection{Relevanz}

Der erste Relevanzindikator ist der Anteil der vorkommenden Suchwörter in Titel und Teasertext. Untersucht wird nur die jeweils erste Seite der Trefferlisten - also die Treffer, die von den Suchmaschinen als die relevantesten eingestuft werden und insgesamt sehr gut zur Suchanfrage passen sollten. Weil für die Nutzer die Anzahl der vorhandenen Suchbegriffe ein „heuristic cue“ bei der Entscheidung über die Relevanz eines Treffers ist (Stark et al. 2014), ist davon auszugehen, dass sie fehlende Suchbegriffe als Zeichen mangelnder Relevanz werten - zumal bei kurzen Suchanfragen aus ein bis zwei Wörtern.

Tabelle 3 zeigt, dass tatsächlich nahezu alle Treffer aller Suchmaschinen sämtliche Suchwörter mindestens einmal in Titel und/oder Teasertext enthalten. Lediglich Ask bietet etwas (aber nicht signifikant) weniger relevante Ergebnisse als die Konkurrenten - wobei auch ein Anteil von 90 noch für Ergebnisse spricht, die sehr gut zur Suchanfrage passen.

Doch auch Treffer, die sämtliche Suchbegriffe enthalten, können unterschiedlich relevant für die Beantwortung der Suchanfrage sein. $\mathrm{Zu}$ vermuten ist, dass Treffer relevanter sind, wenn sie die Suchbegriffe nicht nur aufgreifen, sondern auch in einen logischen Zusammenhang bringen, sodass sich den Nutzern bereits aus den kurzen Titelund Teasertexten der Informationsgehalt des Treffers für die Suche erschließt. Daher 
wird geprüft, zu welchem Anteil die Suchbegriffe in den Treffern unmittelbar zusammenhängen. Auch dieses Kriterium erfüllen sämtliche Suchmaschinen sehr gut (Tabelle $3)$.

Tabelle 3: Relevanz der Trefferlisten

\begin{tabular}{|c|c|c|c|c|c|c|}
\hline & $\begin{array}{l}\text { Google } \\
\text { (A) }\end{array}$ & $\begin{array}{l}\text { Bing } \\
\text { (B) }\end{array}$ & $\begin{array}{l}\text { Ixquick } \\
\text { (C) }\end{array}$ & $\begin{array}{l}\text { Ask } \\
\text { (D) }\end{array}$ & $\begin{array}{l}\text { Lycos } \\
\text { (E) }\end{array}$ & Gesamt \\
\hline $\begin{array}{l}\text { Anteil vorkommender } \\
\text { Suchwörter in Titel \& } \\
\text { Teasertext }\end{array}$ & $\begin{array}{c}.95 \\
(\mathrm{~s}=.17) \\
(\mathrm{n}=66) \\
\end{array}$ & $\begin{array}{c}.98 \\
(s=.12) \\
(n=66) \\
\end{array}$ & $\begin{array}{c}.97 \\
(s=.16) \\
(n=60)\end{array}$ & $\begin{array}{c}.90 \\
(s=.24) \\
(\mathrm{n}=60) \\
\end{array}$ & $\begin{array}{c}.98 \\
(s=.14) \\
(\mathrm{n}=60) \\
\end{array}$ & $\begin{array}{c}.96 \\
(s=.17) \\
(n=312)\end{array}$ \\
\hline $\begin{array}{l}\text { Anteil der zusammen- } \\
\text { hängenden Suchwörter }\end{array}$ & $\begin{array}{c}.98 \\
(\mathrm{~s}=.14) \\
(\mathrm{n}=66) \\
\end{array}$ & $\begin{array}{c}.95 \\
(\mathrm{~s}=.18) \\
(\mathrm{n}=66) \\
\end{array}$ & $\begin{array}{c}.95 \\
(\mathrm{~s}=.18) \\
(\mathrm{n}=60) \\
\end{array}$ & $\begin{array}{c}.92 \\
(\mathrm{~s}=.23) \\
(\mathrm{n}=60) \\
\end{array}$ & $\begin{array}{c}.93 \\
(s=.19) \\
(\mathrm{n}=60) \\
\end{array}$ & $\begin{array}{c}.95 \\
(s=.18) \\
(n=312)\end{array}$ \\
\hline $\begin{array}{l}\text { Inhaltliche Relevanz } \\
(\mathrm{MW})^{1}\end{array}$ & $\begin{array}{c}.95^{\mathrm{D}} \\
(\mathrm{s}=.14) \\
(\mathrm{n}=66)\end{array}$ & $\begin{array}{c}.88 \\
(s=.25) \\
(\mathrm{n}=65)\end{array}$ & $\begin{array}{c}.85 \\
(s=.30) \\
(\mathrm{n}=55)\end{array}$ & $\begin{array}{c}.80 \\
(s=.31) \\
(\mathrm{n}=59)\end{array}$ & $\begin{array}{c}.93 \\
(s=.22) \\
(\mathrm{n}=57)\end{array}$ & $\begin{array}{c}.88 \\
(s=.25) \\
(n=302)\end{array}$ \\
\hline Index Relevanz (MW) ${ }^{1}$ & $\begin{array}{c}.96^{\mathrm{d}} \\
(\mathrm{s}=.10) \\
(\mathrm{n}=66)\end{array}$ & $\begin{array}{c}.94 \\
(s=.14) \\
(\mathrm{n}=65)\end{array}$ & $\begin{array}{c}.94 \\
(s=.13) \\
(\mathrm{n}=55)\end{array}$ & $\begin{array}{c}.87 \\
(\mathrm{~s}=.18) \\
(\mathrm{n}=59)\end{array}$ & $\begin{array}{c}.94 \\
(s=.14) \\
(\mathrm{n}=57)\end{array}$ & $\begin{array}{c}.93 \\
(s=.14) \\
(n=302)\end{array}$ \\
\hline
\end{tabular}

1 Treffer ohne Teasertext werden hier nicht berücksichtigt.

Aa Die Buchstaben hinter den Werten zeigen an, von welchen anderen Werten in derselben Zeile sich der betreffende Wert signifikant unterscheidet (Kleinbuchstaben: $p<0,05$; Großbuchstaben: $\mathrm{p}<0,01)$.

Als dritter Relevanzindikator wird die inhaltliche Relevanz der Treffer für die jeweilige Suchanfrage untersucht. Tabelle 3 weist erneut die Ergebnisse von Google als hochrelevant aus - ein recht stabiler Befund, denn auch die Standardabweichung ist bei Google am geringsten. Von einem großen Vorsprung an inhaltlicher Relevanz kann dennoch keine Rede sein, denn Lycos liegt nur knapp hinter Google, mit etwas Abstand folgen Bing und Ixquick. Signifikant weniger relevant als die Google-Treffer sind nur die von Ask. Zwar deuten die höheren Standardabweichungen insbesondere bei Ask und $I x$ quick darauf hin, dass auch diese beiden Suchmaschinen teilweise hochrelevante Ergebnisse liefern; die Nutzer können sich aber weniger darauf verlassen als bei Google.

Fasst man die drei Relevanzindikatoren zu einem Index zusammen, kann F1b folgendermaßen beantwortet werden: Google, Lycos, Bing und Ixquick und liefern Treffer, die sehr gut zur Suchanfrage passen. Etwas weniger relevant sind die Suchergebnisse von Ask, von einem Relevanzdefizit kann angesichts des immer noch sehr hohen Indexwertes aber nicht gesprochen werden.

\subsection{Gattungsvielfalt}

Die Gattungsvielfalt wird anhand der Verschiedenartigkeit der Treffergattungen gemessen und durch das Maß der relativen Entropie operationalisiert. Tabelle 4 zeigt, dass sie bei allen fünf Suchmaschinen relativ hoch ist, dass also alle den Nutzern ein relativ breites Spektrum unterschiedlicher Treffergattungen präsentieren. Am häufigsten verweisen die Treffer wie bei Beiler (2013, S. 224) auf die Internetseiten klassischer Medienanbieter, wobei Printmedien (insbesondere namhafte, überregionale) deutlich häufiger vertreten 
sind als Rundfunkveranstalter. Am häufigsten wird auf Spiegel Online (21 von 95 Printmedien-Treffern) und die Süddeutsche Zeitung (14 Treffer) verlinkt.

Tabelle 4: Gattungsvielfalt der Trefferlisten

\begin{tabular}{|c|c|c|c|c|c|c|}
\hline & $\begin{array}{l}\text { Google } \\
\text { (A) } \\
\%\end{array}$ & $\begin{array}{c}\text { Bing } \\
\text { (B) } \\
\%\end{array}$ & $\begin{array}{c}\text { Ixquick } \\
\text { (C) } \\
\%\end{array}$ & $\begin{array}{l}\text { Ask } \\
\text { (D) } \\
\%\end{array}$ & $\begin{array}{c}\text { Lycos } \\
(\mathrm{E}) \\
\%\end{array}$ & $\begin{array}{l}\text { Gesamt } \\
\%\end{array}$ \\
\hline $\begin{array}{l}\text { Internetableger eines } \\
\text { klassischen Mediums } \\
\text { Seite eines Verbands/ }\end{array}$ & 53 & 41 & 29 & 27 & 32 & 37 \\
\hline $\begin{array}{l}\text { Vereins/ öffentl. Ein- } \\
\text { richtung }\end{array}$ & 17 & 23 & 32 & 37 & 28 & 27 \\
\hline Portale & 9 & 18 & 15 & 10 & 7 & 12 \\
\hline $\begin{array}{l}\text { Social Media/ Seite } \\
\text { einer Privatperson }\end{array}$ & 3 & 8 & 5 & 5 & 18 & 8 \\
\hline Wikipedia & 8 & 9 & 9 & 2 & 10 & 7 \\
\hline $\begin{array}{l}\text { Seiten kommerzieller } \\
\text { Unternehmen }\end{array}$ & 5 & 2 & 5 & 13 & 2 & 5 \\
\hline Reines Onlinemedium & 6 & 0 & 3 & 5 & 3 & 4 \\
\hline $\begin{array}{l}\text { Suchmaschine/ Link- } \\
\text { sammlung }\end{array}$ & 0 & 0 & 2 & 2 & 0 & 1 \\
\hline Gesamt & $\begin{array}{c}100 \\
(n=66)\end{array}$ & $\begin{array}{c}100 \\
(n=66)\end{array}$ & $\begin{array}{c}100 \\
(n=59)\end{array}$ & $\begin{array}{c}100 \\
(n=60)\end{array}$ & $\begin{array}{c}100 \\
(n=60)\end{array}$ & $\begin{array}{c}100 \\
(n=311)\end{array}$ \\
\hline $\begin{array}{l}\text { Index Gattungsvielfalt } \\
\text { (relative Entropie) }^{1}\end{array}$ & .63 & $.65^{\mathrm{A}}$ & $.75^{\mathrm{A}, \mathrm{B}, \mathrm{E}}$ & .79 A,B,C,E & $.73^{\mathrm{A}, \mathrm{B}}$ & .71 \\
\hline
\end{tabular}

${ }^{1}$ Für die vorliegende Darstellung wurden einzelne Gattungen zusammengefasst. Die Gattungsentropie wurde auf Basis der differenzierteren Verteilung berechnet. Da die Berechnungen auf Basis der einzelnen Treffer erfolgen, wurde allen Treffern einer Suchmaschine der entsprechende Wert zugewiesen. Die Standardabweichung nimmt somit den Wert 0 an. Das erklärt, warum selbst kleine Differenzen zwischen den Suchmaschinen signifikant sind. Die Signifikanzen sollten daher vorsichtig interpretiert werden. Abweichungen von $100 \%$ in den Spaltensummen ergeben sich durch Rundungsfehler.

Ask weist die vielfältigsten Treffergattungen und zudem eine andere Verteilung als die anderen Suchmaschinen auf: Es entfallen weniger Treffer auf Online-Ableger klassischer Massenmedien, dafür aber deutlich mehr auf eher seltene Gattungen wie z. B. reine Online-Medien oder kommerzielle Seiten. Dem Vielfaltsindex zufolge ist das Spektrum unterschiedlicher Treffergattungen bei allen anderen Suchmaschinen weniger breit, bei Bing und Google sogar signifikant weniger breit als bei $A s k$, was an deren relativ starker Konzentration auf Nachrichtenseiten liegt. Somit kann F1c wie folgt beantwortet werden: Alle Suchmaschinen sind - zumindest auf ihren ersten Trefferlistenseiten - deutlich von der größtmöglichen Gattungsvielfalt entfernt. Bei Google und Bing ist diese sogar noch stärker eingeschränkt als bei den drei Mitbewerbern.

\subsection{Aktualität}

Auch hinsichtlich ihrer Aktualität unterscheiden sich die fünf Suchmaschinen recht deutlich (Tabelle 5). Zunächst fällt auf, dass der Anteil der Treffer, die überhaupt eine 
Datumsangabe enthalten, insgesamt sehr gering ist. Zwar bedeutet eine fehlende Datumsangabe nicht zwangsläufig, dass ein Treffer nicht aktuell ist, und bei den hier betrachteten „Dauerbrennerthemen“ ist Tagesaktualität nicht zwingend erforderlich, insbesondere bei Informationsquellen wie Einträgen in Wikis oder Informationen auf Unternehmensseiten. Deshalb verwundert es auch nicht, dass die Treffer, die ein Datum enthalten, insgesamt nicht sehr aktuell sind. Hierin kommt ein Spezifikum des Internets zum Ausdruck: Im Unterschied zu den traditionellen Massenmedien erfüllt es auch eine Archivfunktion, für die das Kriterium der zeitlichen Aktualität keine Rolle spielt. Bei tagesaktuellen Themen würde dies mehr ins Gewicht fallen, dort wären vermutlich aber auch häufiger Datumsangaben vorhanden. Weil Datumsangaben jedoch für viele Nutzer ein heuristischer Schlüsselreiz zur Selektion relevanter Treffer sind (Stark et al. 2014), ist deren Fehlen aus ihrer Sicht als Qualitätsdefizit zu werten - das bei den fünf Suchmaschinen unterschiedlich stark ausgeprägt ist.

Während bei Google rund die Hälfte und bei Ask etwa ein Drittel der Treffer ein Datum enthält, sind es bei den anderen Suchmaschinen maximal zehn Prozent. Der Aktualitätsvorsprung von Google setzt sich darin fort, dass der Marktführer (abgesehen von drei Treffern bei Bing) als einzige Suchmaschine zumindest wochenaktuelle Suchergebnisse bietet. Das dürfte mit dem oben erwähnten hohen Anteil von tagesaktuellen Nachrichtenseiten an den Google-Trefferlisten zusammenhängen - wobei in Beilers (2013, S. 379) Studie sogar bei Nachrichtensuchmaschinen, für die Aktualität noch wichtiger ist, die verlinkten Beiträge im Schnitt fast 1,5 Tage alt sind. Auch bei Nachrichtenseiten zeigt Google einer detaillierteren Auswertung zufolge häufiger ein Datum an als die anderen Suchmaschinen, bietet also qualitativ hochwertigere Trefferlisten. Die Antwort auf F1d lautet demnach: Auch bei der Aktualität der Trefferlisten liegt Google signifikant vor der Konkurrenz. Zwar ist der Indexwert von .29 recht gering, die vier Mitbewerber schneiden aber noch schlechter ab.

Tabelle 5: Aktualität der Trefferlisten

\begin{tabular}{|c|c|c|c|c|c|c|}
\hline & $\begin{array}{c}\text { Google } \\
\text { (A) } \\
\%\end{array}$ & $\begin{array}{l}\text { Bing } \\
\text { (B) } \\
\%\end{array}$ & $\begin{array}{c}\text { Ixquick } \\
\text { (C) } \\
\%\end{array}$ & $\begin{array}{l}\text { Ask } \\
\text { (D) } \\
\%\end{array}$ & $\begin{array}{c}\text { Lycos } \\
(\mathrm{E}) \\
\%\end{array}$ & $\begin{array}{c}\text { Gesamt } \\
\%\end{array}$ \\
\hline Am selben Tag & 12 & 5 & 0 & 0 & 0 & 4 \\
\hline In derselben Woche & 9 & 0 & 0 & 0 & 0 & 2 \\
\hline $\begin{array}{l}\text { Im selben Monat und } \\
\text { Vormonat }\end{array}$ & 9 & 5 & 0 & 18 & 0 & 6 \\
\hline Älter als Vormonat & 21 & 2 & 2 & 13 & 2 & 8 \\
\hline Kein Datum angegeben & 49 & 89 & 98 & 68 & 98 & 80 \\
\hline Gesamt & $\begin{array}{c}100 \\
(n=66)\end{array}$ & $\begin{array}{c}100 \\
(n=66)\end{array}$ & $\begin{array}{c}100 \\
(\mathrm{n}=60)\end{array}$ & $\begin{array}{c}100 \\
(n=60)\end{array}$ & $\begin{array}{c}100 \\
(\mathrm{n}=60)\end{array}$ & $\begin{array}{c}100 \\
(\mathrm{n}=312)\end{array}$ \\
\hline Index Aktualität (MW) & $\begin{array}{c}.29 \mathrm{~B}, \mathrm{C}, \mathrm{d}, \mathrm{E} \\
(\mathrm{s}=.36) \\
(\mathrm{n}=66)\end{array}$ & $\begin{array}{c}.07 \\
(s=.23) \\
(n=66)\end{array}$ & $\begin{array}{c}.00 \\
(s=.03) \\
(n=60)\end{array}$ & $\begin{array}{l}.13 \mathrm{C}, \mathrm{E} \\
(\mathrm{s}=.20) \\
(\mathrm{n}=60)\end{array}$ & $\begin{array}{c}.00 \\
(s=.03) \\
(n=60)\end{array}$ & $\begin{array}{c}.10 \\
(s=.24) \\
(n=312)\end{array}$ \\
\hline
\end{tabular}

Aa Die Buchstaben hinter den Werten zeigen an, von welchen anderen Werten in derselben Zeile sich der betreffende Wert signifikant unterscheidet (Kleinbuchstaben: $\mathrm{p}<0,05$; Großbuchstaben: $\mathrm{p}<0,01)$. Abweichungen von $100 \%$ in den Spaltensummen ergeben sich durch Rundungsfehler. 


\subsection{Gesamtqualität der Suchmaschinen}

Um die Qualität der Trefferlisten insgesamt gemäß F1 beurteilen zu können, werden die vier Teilindizes abschließend zu einem Gesamtindex zusammengefasst (Tabelle 6). Wie schon bei mehreren Qualitätskriterien weist Google wenig überraschend auch insgesamt die höchste Qualität auf, gefolgt - allerdings mit deutlichem Abstand - vom größten Konkurrenten Bing. Noch größer ist der Abstand zu Ixquick, Ask und Lycos. Googles signifikanter Gesamtvorsprung begründet sich vor allem durch eine konstant hohe Qualität über die einzelnen Kriterien hinweg: Während die meisten anderen Suchmaschinen bei manchen Kriterien besser, bei anderen schlechter abschneiden, landet Google fast durchgängig auf den vordersten Rängen. ${ }^{12}$ Bei der Aktualität liegt das allerdings nicht an einer guten Leistung von Google, sondern an der noch schlechteren Leistung der Konkurrenten. Das einzige Kriterium, bei dem der Marktführer hinter den anderen Anbietern zurückbleibt, ist die Gattungsvielfalt.

Tabelle 6: Gesamtqualität der Trefferlisten

\begin{tabular}{ccccccc}
\hline & $\begin{array}{c}\text { Google } \\
(\mathrm{A})\end{array}$ & $\begin{array}{c}\text { Bing } \\
(\mathrm{B})\end{array}$ & $\begin{array}{c}\text { Ixquick } \\
(\mathrm{C})\end{array}$ & $\begin{array}{c}\text { Ask } \\
(\mathrm{D})\end{array}$ & $\begin{array}{c}\text { Lycos } \\
(\mathrm{E})\end{array}$ & Gesamt \\
\hline \multirow{3}{*}{ Index Qualität gesamt } & $.56^{\mathrm{B}, \mathrm{C}, \mathrm{D}, \mathrm{E}}$ & $.41^{\mathrm{c}, \mathrm{D}, \mathrm{E}}$ & $.36^{\mathrm{E}}$ & .33 & .31 & .40 \\
& $(\mathrm{~s}=.16)$ & $(\mathrm{s}=.13)$ & $(\mathrm{s}=.06)$ & $(\mathrm{s}=.09)$ & $(\mathrm{s}=.06)$ & $(\mathrm{s}=.14)$ \\
& $(\mathrm{n}=66)$ & $(\mathrm{n}=65)$ & $(\mathrm{n}=55)$ & $(\mathrm{n}=59)$ & $(\mathrm{n}=57)$ & $(\mathrm{n}=302)$ \\
\hline
\end{tabular}

Aa Die Buchstaben hinter den Werten zeigen an, von welchen anderen Werten in derselben Zeile sich der betreffende Wert signifikant unterscheidet (Kleinbuchstaben: $\mathrm{p}<0,05$; Großbuchstaben: $\mathrm{p}<0,01)$.

\section{Diskussion}

Die Marktdominanz von Google, die Gatekeeper-Position von Suchmaschinen im Internet und ihre daraus resultierende gesellschaftliche Verantwortung, geben Anlass zur Frage nach deren Qualität. Die vorliegende Studie untersucht die Qualität der Trefferlisten von fünf Suchmaschinen anhand von vier Kriterien: Benutzerfreundlichkeit, Relevanz, Gattungsvielfalt und Aktualität. Sie prüft, ob die Suchmaschinen die Voraussetzungen dafür bieten, dass die Nutzer zwei Rollen erfüllen können - die des Konsumenten, für den der Nutzwert im Fokus steht, und die des Bürgers, der sich an gesellschaftlichen Wertvorstellungen orientiert.

Aus der Konsumentenperspektive schneiden die Suchmaschinen unterschiedlich gut ab. Während alle fünf hochrelevante Ergebnisse erbringen, ihrer Kernfunktion also sehr gut nachkommen, unterscheiden sie sich bei der Benutzerfreundlichkeit erkennbar: Hier liegt Google klar vor Bing, Ixquick, Lycos und Ask. Dieser Befund deutet in dieselbe Richtung wie Nutzerbefragungen, denen zufolge die Nutzer als Konsumenten Google für die beste Suchmaschine halten (Stark et al. 2014).

Etwas anders fällt die Bewertung aus der Bürgerperspektive aus. Die Gattungsvielfalt ist das einzige Kriterium, bei dem Google nicht vor der Konkurrenz liegt. Da dies auf

12 Das gilt auch, wenn man die Ergebnisse nach den sechs Suchthemen differenziert betrachtet. Unterschiede gibt es nur beim Abstand von Google zur Konkurrenz: Bei „Stress Schulkinder" liegen Google, Bing und Ixquick, bei „Mindestlohn“ Google und Bing recht nah beieinander, doch bei allen anderen Themen ist der Vorsprung von Google vor sämtlichen Konkurrenten deutlich. 
Googles relativ starke Konzentration auf massenmediale Treffer zurückzuführen ist, könnte dieses vermeintliche Defizit aber auch ein Vorteil sein: Zum einen geht es mit einem Aktualitätsvorsprung einher, was den inhärenten Widerspruch zwischen verschiedenen Qualitätskriterien deutlich macht: Alle gleichzeitig optimal zu erfüllen, ist schlichtweg nicht möglich. Es kommt also darauf an, allen Qualitätsansprüchen bis zu einem gewissen Grad zu entsprechen und nirgends zu große Abstriche zu machen. ${ }^{13}$ Zum anderen ist denkbar, dass gerade die Konzentration auf massenmediale Inhalte dazu führt, dass die Nutzer mit qualitätsvolleren Inhalten in Berührung kommen. Die wenigen vorhandenen Studien zum Vergleich der Qualität massenmedialer und anderer Internetinhalte deuten zumindest darauf hin, dass erstere vielfältiger sind (Neuberger \& Lobigs 2010; Oschatz et al. 2014).

Zudem besagt der Befund nur, dass der Marktführer strukturell weniger vielfältig ist als die Konkurrenz. Bedenklicher wären Defizite bei der inhaltlichen Vielfalt, etwa ein Fokus auf ganz bestimmte Sichtweisen. Ob das der Fall ist, wie von einigen Autoren befürchtet (Dörr \& Schuster 2014; Pariser 2011), muss vorerst jedoch offen bleiben. Hierzu braucht es weiterführende Studien, die sich nicht auf die Trefferlisten beschränken, sondern auch die darauf verlinkten Websites analysieren. Mit Blick auf die Bürgerperspektive sollte dabei insbesondere auch die gesellschaftliche Relevanz der Suchergebnisse betrachtet werden.

Dass es Google offenbar besonders gut gelingt, die Bedürfnisse der Nutzer in ihrer Konsumentenrolle zu befriedigen, könnte die klaren Nutzerpräferenzen für diese Suchmaschine zumindest teilweise begründen. Dass die daraus resultierende Dominanz in Zukunft weiter bestehen wird, ist sehr wahrscheinlich. Die Suchmaschinennutzung ist mittlerweile hochgradig habitualisiert (van Deursen et al. 2011) - und wenn sich die Nutzer erst einmal auf eine Suchmaschine festgelegt haben, mit ihr zufrieden sind, der Marke Loyalität entgegenbringen und eine emotionale Bindung zu ihr aufgebaut haben, wird es für alternative Angebote sehr schwer, deren Vormachtstellung zu durchbrechen (Veloutsou und McAlonan 2012).

Weiter verstärkt wird Googles Dominanz einerseits dadurch, dass die Zahl der mit einer Suchmaschine getätigten Suchen die Qualität der Ergebnisse beeinflusst, andererseits durch sogenannte Lock-in-Effekte: Beschränkte sich Googles Leistung auf die Suchmaschinenfunktion, wären die Hürden für einen Wechsel zu anderen Suchmaschinen relativ gering (Schulz et al. 2011, S. 17). Der Konzern ergänzt seine Suchmaschine aber durch eine breit gefächerte Palette verschiedenster, miteinander verknüpfter Dienste - etwa den Webmail-Dienst Gmail, den Browser Chrome und das Handy-Betriebssystem Android - und bindet die Nutzer dadurch immer enger an sich. Je mehr dieser Dienste die Rezipienten nutzen, desto besser kann das Angebot jedes einzelnen Dienstes auf ihre individuellen Bedürfnisse zugeschnitten werden. Das macht die Nutzung immer einfacher und bequemer, entspricht also den Kernbedürfnissen der Konsumenten. Die Schwelle für einen Wechsel zu anderen Anbietern wächst dadurch stetig, und mit ihr das Einflusspotenzial von Google - auch aufgrund der stark heuristischen Nutzungsweise von Suchmaschinen: Je einfacher, intuitiver und bequemer die Nutzer ans Ziel ihrer Suche kommen, desto weniger Anlass haben sie, sich kritisch mit der Suchmaschine und ihren Trefferlisten auseinanderzusetzen. Benutzerfreundlich gestaltete Suchmaschinen

13 Beiler (2013) zeigt Qualitätsdefizite auch für Nachrichtensuchmaschinen. Seine Ergebnisse sind mit denen der vorliegenden Studie jedoch nur eingeschränkt vergleichbar, weil er größtenteils andere (bzw. anders operationalisierte) Qualitätskriterien und keine Universalsuchmaschinen untersucht. Die Ergebnisse von Machill et al. (2003) lassen sich aufgrund der unterschiedlichen Zusammensetzung des Samples schlecht mit den vorliegenden vergleichen. 
begünstigen also die ohnehin unbewusste und unkritische Nutzung noch weiter. Damit steht die aus der Konsumentensicht begrüßenswerte Benutzerfreundlichkeit der Ausübung der Bürgerrolle eher entgegen als sie zu fördern.

Die vorliegende Analyse zeigt, dass die Differenzierung von Nutzerrollen sinnvoll ist, um die Qualität von Suchmaschinen umfassend zu untersuchen, und kann damit als Ausgangspunkt weiterführender Studien dienen. Neben der bereits erwähnten Erfassung der inhaltlichen Vielfalt und gesellschaftlichen Relevanz der Treffer sollten künftige Inhaltsanalysen auch das Themenspektrum durch eine größere Anzahl an Suchanfragen erweitern, orientiert an der Retrieval-Forschung, die mittlerweile mit einem Minimum von 50 Suchanfragen arbeitet (Lewandowski 2015, S. 218). Zudem sollte die Qualität von Suchmaschinen aus der (hier ausgeblendeten) Perspektive des Nutzers als Rechteinhaber untersucht werden. Viele Nutzer fürchten etwa, dass Suchmaschinen ihre Schutzbedürfnisse - insbesondere den Datenschutz - verletzen (Stark et al. 2014). Um das tatsächliche Ausmaß dieser Bedrohung abschätzen zu können, braucht es Studien, die über Inhaltsanalysen hinausgehen.

Die rasanten und stetigen Weiterentwicklungen der Suchmaschinentechnologie unterstreichen die Notwendigkeit einer fortlaufenden Beschäftigung mit der Qualität von Suchmaschinen. Im April 2015 hat Google bekanntgegeben, in einer Kooperation mit acht Verlagen aus mehreren europäischen Ländern - darunter z. B. die FAZ, Die Zeit, The Guardian, El Pais und Les Echos - „Schulungen, Forschung und technische Lösungen für den digitalen Journalismus vorantreiben“ (Tieschky 2015) zu wollen. Die sogenannte „Digital News Initiative“ zielt darauf ab, die Kritiker in der eingangs erwähnten Debatte um eine Regulierung des Suchmaschinenmarktes zu besänftigen (ebd.). Allerdings könnte dies die Unabhängigkeit des Journalismus gefährden und eine weitere Konzentration der Presselandschaft nach sich ziehen (Amjahid 2015). Um die Auswirkungen und potenzielle Bedrohungen durch solche Entwicklungen besser einschätzen und ihnen entgegenwirken zu können, muss die Qualität von Suchmaschinen kontinuierlich weiter erforscht werden. Die vorliegende Studie stellt dafür ein auf vielfältige Themenbereiche anwendbares Instrumentarium bereit.

\section{Literatur}

Amjahid, M. (2015). Google und deutsche Zeitungen - was soll das? http://www.tagesspiegel.de/ medien/digital-news-initiative-google-und-deutsche-zeitungen-was-soll-das/11738598.html [29.09.2015].

Arnold, K. (2006). Publikumsorientierte Qualität - ein Weg aus der Zeitungskrise?, in: S. Weischenberg, W. Loosen, \& M. Beuthner (Hrsg.), Medien-Qualitäten. Öffentliche Kommunikation zwischen ökonomischem Kalkül und Sozialverantwortung (S. 415-434). Konstanz: UVK.

Arnold, K. (2009). Qualitätsjournalismus. Die Zeitung und ibr Publikum. Konstanz: UVK.

Atkin, C. (1973). Instrumental Utilities and Information Seeking, in: P. Clarke (Ed.), New Models for Mass Communication (S. 205-242). Beverly Hills: Sage.

Bar-Ilan, J., Keenoy, K., Yaari, E., \& Levene, M. (2007). User Rankings of Search Engine Results. Journal of the American Society for Information Science and Technology, 58(9), 1254-1266.

Beiler, M. (2013). Nachrichtensuche im Internet. Inhaltsanalyse zur journalistischen Qualität von Nachrichtensuchmaschinen. Konstanz: UVK.

ComScore (2013). Future in Focus: Digitales Deutschland 2013. http://www.comscore.com/ger/ Insights/Praesentationen-und-Whitepapers/2013/2013-Future-in-Focus-Digitales-Deutsch land [21.09.2015].

Deka, S. K., \& Lahkar, N. (2010). Performance Evaluation and Comparison of the Five Most Used Search Engines in Retrieving Web Resources. Online Information Review, 34(5), 757-771.

Dörr, D., \& Schuster, S. (2014). Suchmaschinen im Spannungsfeld zwischen Nutzung und Regulierung. Rechtliche Bestandsaufnahme und Grundstrukturen einer Neuregelung, in: B. Stark, 
D. Dörr, \& S. Aufenanger (Hrsg.), Die Googleisierung der Informationssuche. Suchmaschinen zwischen Nutzung und Regulierung. (S. 262-323). Berlin: de Gruyter.

Franzmann, G., \& Wagner, M. (1999). Heterogenitätsindizes zur Messung der Pluralität von Lebensformen und ihre Berechnung in SPSS. ZA-Information (44), 75-95.

Google (2013). Alles über die Suche. http://www.google.com/intl/de_ALL/insidesearch/ [29.09.2015].

Hagen, L. M. (1995). Relevanz von Nachrichten. Meßmethoden für ein zentrales Qualitätskriterium und ihre Anwendung auf Dienste von Nachrichtenagenturen. Rundfunk und Fernseben, 43(2), 158-177.

Hasebrink, U. (2012). The Role of the Audience within Media Governance: the Neglected Dimension of Media Literacy. Media Studies, 23(6), 58-73.

Hasebrink, U. (18. Juni 2013). Warum Qualität ein Thema ist. Präsentation auf dem Symposium „(Wie) Verändert sich Qualität? Transformation durch Digitalisierung; Anspruchshaltung, Wettbewerbsfähigkeit und Positionierung des Rundfunks“ der ARD/ZDF Medienakademie und des Instituts für Rundfunktechnik München, 18. Juni 2013, http://www.medienpolitik.net/ wp-content/uploads/2013/07/2013-06-18-ARD-ZDF-Medienakademie-Qualit\%C3 \% A4t. pdf [29.09.2015].

Hasebrink, U., \& Herzog, A. (2009). Die Sicht der Zuschauer. Vorstudie zu einem regelmäßigen Monitoring der wahrgenommenen Qualitäten des Fernsehens. http://www.hans-bredow-in stitut.de/webfm_send/660 [21.09.2015].

Hasebrink, U., \& Schmidt, J.-H. (2013). Medienübergreifende Informationsrepertoires. Zur Rolle der Mediengattungen und einzelner Angebote für Information und Meinungsbildung. Media Perspektiven (1), 2-12.

Heuzeroth, T. (2014). EU-Parlament fordert die Zerschlagung von Google. http://www.welt.de/ wirtschaft/article134790642/EU-Parlament-fordert-die-Zerschlagung-von-Google.html [21.09.2015].

Höchstötter, N., \& Koch, M. (2009). Standard Parameters for Searching Behaviour in Search Engines and their Empirical Evaluation. Journal of Information Science, 35(1), 45-65.

ixquick.com (2013). Ixquick schützt Ihre Privatsphäre! https://ixquick.com/deu/protect-priva cy.html [21.09.2015].

Latzer, M., Hollnbuchner, K., Just, N., \& Saurwein, F. (2014). The Economics of Algorithmic Selection on the Internet. http://www.mediachange.ch/media/pdf/publications/Economics _of_algorithmic_selection_WP.pdf [21.09.2015].

Lewandowski, D. (2008). The Retrieval Effectiveness of Web Search Engines: Considering Results Descriptions. Journal of Documentation, 64(6), 915-937.

Lewandowski, D. (2013). Verwendung von Skalenbewertungen in der Evaluierung von Web-Suchmaschinen, in: H.-C. Hobohm (Hrsg.), Informationswissenschaft zwischen virtueller Infrastruktur und materiellen Lebenswelten. Tagungsband des 13. Internationalen Symposiums für Informationswissenschaft (ISI 2013), Potsdam, 19.-22. März 2013 (S. 339-348). Glückstadt: Werner Hülsbusch.

Lewandowski, D. (2015). Suchmaschinen verstehen. Berlin, Heidelberg: Springer Vieweg.

Machill, M., Lewandowski, D., \& Karzauninkat, S. (2005). Journalistische Aktualität im Internet. Ein Experiment mit den „News-Suchfunktionen“ von Suchmaschinen, in: M. Machill, \& N. Schneider (Hrsg.), Suchmaschinen: Neue Herausforderungen für die Medienpolitik (S. 105164). Berlin: Vistas.

Machill, M., Neuberger, C., Schweiger, W., \& Wirth, W. (2003). Wegweiser im Netz: Qualität und Nutzung von Suchmaschinen, in: M. Machill, \& C. Welp (Hrsg.), Wegweiser im Netz. Qualität und Nutzung von Suchmaschinen (S. 13-490). Gütersloh: Verlag Bertelsmann-Stiftung.

Mehlis, K. (2014). Von der Sender- zur Nutzerqualität. Entwicklung einer mehrdimensionalen Skala zur Messung der Qualität von Online-Nachrichtenangeboten aus Publikumssicht, in: W. Loosen, \& M. Dohle (Hrsg.), Journalismus und (sein) Publikum. Schnittstellen zwischen Journalismusforschung und Rezeptions- und Wirkungsforschung (S. 253-271). Wiesbaden: Springer VS.

Mill, J. S. (1956). On Liberty. New York: The Liberal Arts Press. 
Mizzarro, S. (1997). Relevance: The Whole History. Journal of the American Society for Information Science and Technology, 48(9), 810-832.

Neuberger, C. (2005). Angebot und Nutzung von Internet-Suchmaschinen. Marktstrategien, Qualitätsaspekte, Regulierungsziele. Media Perspektiven (1), 2-13.

Neuberger, C. (2011). Definition und Messung publizistischer Qualität im Internet. Herausforderungen des Drei-Stufen-Tests. Berlin: Vistas.

Neuberger, C. (2012). Journalismus im Internet aus Nutzersicht. Ergebnisse einer Onlinebefragung. Media Perspektiven (1), 40-55.

Neuberger, C., \& Lobigs, F. (2010). Die Bedeutung des Internets im Rahmen der Vielfaltssicherung. Gutachten im Auftrag der Kommission zur Ermittlung der Konzentration im Medienbereich $(K E K)$. Berlin: Vistas.

O'Neill, S. (2013). Hier wurden selbst Hunde geopfert. Von MySpace zu Palm. http://www. pcwelt.de/ratgeber/Von_MySpace_zu_Palm-Hier_wurden_selbst_Hunde_geopfert-7917506. html [29.09.2015].

Oschatz, C., Maurer, M., \& Haßler, J. (2014). (R)Evolution der Politikberichterstattung im Medienwandel? Die Inhalte von nachrichtenjournalistischen Online- und Offline-Angeboten im Vergleich. Medien E Kommunikationswissenschaft, 62(1), 25-41.

Pariser, E. (2011). The Filter Bubble: What the Internet Is Hiding from You. Penguin Press: New York.

Presse- und Informationsamt der Bundesregierung (2015). Moderne Medienregulierung - Bund und Länder einigen sich auf Schwerpunktthemen für die Arbeit der Bund-Länder-Kommission zur Medienkonvergenz. http://www.bundesregierung.de/Content/DE/Pressemitteilungen/ BPA/2015/03/2015-03-26-bkm-medienkonvergenz.html [21.09.2015].

Purcell, K., Brenner, J., \& Rainie, L. (2012). Search Engine Use 2012. http://www.pewinternet.org/ 2012/03/09/search-engine-use-2012 [01.10.2015].

Quirmbach, S. (2012). Suchmaschinen: User Experience, Usability und nutzerzentrierte WebsiteGestaltung. Berlin, Heidelberg: Springer Vieweg.

Röhle, T. (2010). Der Google-Komplex. Über Macht im Zeitalter des Internets. Bielefeld: transcript.

Rössler, P., \& Wirth, W. (2001). Inhaltsanalysen im World Wide Web. Probleme und Perspektiven, in: W. Wirth, \& E. Lauf (Hrsg.), Inhaltsanalyse: Perspektiven, Probleme, Potentiale (S. 280302). Köln: Halem.

Ruß-Mohl, S. (1992). Am eigenen Schopfe ... Qualitätssicherung im Journalismus - Grundfragen, Ansätze, Näherungsversuche. Publizistik, 37, 83-96.

Saracevic, T. (2007). Relevance: A Review of the Literature and a Framework for Thinking on the Notion in Information Science. Part II: Nature and Manifestations of Relevance. Journal of the American Society for Information Science and Technology, 58(13), 1915-1933.

Schatz, H., \& Schulz, W. (1992). Qualität von Fernsehprogrammen. Kriterien und Methoden zur Beurteilung von Programmqualität im dualen Fernsehsystem. Media Perspektiven (11), 690712.

Schulz, W., Dreyer, S., \& Hagemeier, S. (2011). Medienpolitik. Machtverschiebung in der öffentlichen Kommunikation. Bonn: Friedrich-Ebert-Stiftung.

Selbstkontrolle Suchmaschinen (2004). Verhaltenssubkodex für Suchmaschinenanbieter der FSM (VK-S). https://www.fsm.de/selbstverpflichtungen/suchmaschinen/Verhaltenssubkodex_ SuMa_VKS_final_20040221_de.pdf [21.09.2015].

Stark, B. (2014). „Don't be evil“. Die Macht von Google und die Ohnmacht der Nutzer und Regulierer, in: B. Stark, D. Dörr, \& S. Aufenanger (Hrsg.), Die „Googleisierung “ der Informationssuche. Suchmaschinen zwischen Nutzung und Regulierung (S. 1-19). Berlin: de Gruyter.

Stark, B., Magin, M., \& Jürgens, P. (2014). Navigieren im Netz - Befunde einer qualitativen und quantitativen Nutzerbefragung, in: B. Stark, D. Dörr, \& S. Aufenanger (Hrsg.), Die „ Googleisierung" der Informationssuche. Suchmaschinen zwischen Nutzung und Regulierung (S. 20-74). Berlin: de Gruyter.

Tieschky, C. (2015). Google sucht neue Freunde. http://www.sueddeutsche.de/medien/googlekooperiert-mit-verlagen-auf-der-suche-nach-neuen-freunden-1.2457127 [29.09.2015]. 
van Deursen, Alexander J. A. M., van Dijk, Jan A. G. M., \& Peters, O. (2011). Rethinking Internet Skills. The Contribution of Gender, Age, Education, Internet Experience, and Hours Online to Medium- and Content-Related Internet Skills. Poetics, 39(2), 125-144.

van Eimeren, B. (2015). Nachrichtenrezeption im Internet. Befunde aus der ARD/ZDF-Onlinestudie 2014. Media Perspektiven (1), 2-7.

Veloutsou, C., \& McAlonan, A. (2012). Loyalty and or Disloyalty to a Search Engine: the Case of Young Millennials. Journal of Consumer Marketing, 29(2), 125-135.

Wäscher, T. (2015). Google Inc. http://www.mediadb.eu/datenbanken/internationale-medien konzerne/google-inc.html [21.09.2015].

Webster, J. G., \& Phalen, P. F. (1994). Victim, Consumer, or Commodity? Audience Models in Communication Policy, in: J. S. Ettema, \& D. C. Whitney (Hrsg.), Audiencemaking. How the Media Create the Audience (S. 19-37). London: Sage.

Weßler, H., \& Rinke, E. M. (2014). Deliberative Performance of Television News in Three Types of Democracy: Insights from the United States, Germany, and Russia. Journal of Communication, 64(5), 827-851.

Wirth, W., Böcking, T., Karnowski, V., \& Pape, T. von. (2007). Heuristic and Systematic Use of Search Engines. Journal of Computer-Mediated Communication, 12(3), 778-800.

Zerback, T. (2013). Publizistische Vielfalt. Demokratischer Nutzen und Einflussfaktoren. Konstanz: UVK.

\section{Das Geschäftsmodell „Paid Content“}

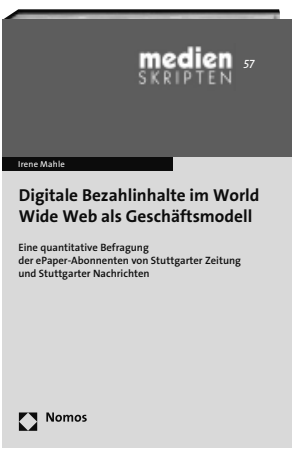

\section{Digitale Bezahlinhalte im World Wide Web als Geschäftsmodell}

Eine quantitative Befragung der ePaper-Abonnenten von Stuttgarter Zeitung und Stuttgarter Nachrichten Von Irene Mahle 2016, ca. 120 S., brosch., ca. 24,- $€$ ISBN 978-3-8487-2463-5

(medien Skripten, $B d$. 57)

Erscheint ca. Januar 2016 www.nomos-shop.de/26147

Wie können Tageszeitungsverlage im Internet gewinnbringend Inhalte vermarkten? Vor diesem Kontext werden auf theoretischer und empirischer Basis das Geschäftsmodell „Paid Content“ analysiert sowie Erfolgsfaktoren bestimmt, die für die

\section{Portofreie Buch-Bestellungen unter} www.nomos-shop.de

Alle Preise inkl. Mehrwertsteuer

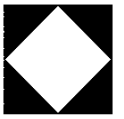

Nomos 